\title{
Potential Therapeutic Agents, Polymethoxylated Flavones Isolated from Kaempferia parviflora for Cataract Prevention through Inhibition of Matrix Metalloproteinase-9 in Lens Epithelial Cells
}

\author{
Yoshiki Miyata, ${ }^{a}$ Jin Tatsuzaki, ${ }^{b}$ Jinwei Yang, ${ }^{b}$ and Hiroshi Kosano ${ }^{*, a}$ \\ ${ }^{a}$ Faculty of Pharma-Sciences, Teikyo University; 2-11-1 Kaga, Itabashi-ku, Tokyo 173-8605, Japan: and ${ }^{b}$ Tokiwa \\ Phytochemical Co., Ltd.; 158 Kinoko, Sakura, Chiba 285-0801, Japan. \\ Received March 15, 2019; accepted July 25, 2019
}

Natural flavonoids have powerful antioxidant activity and have been reported to show promising protective effects against cataracts. The plant Kaempferia parviflora (K. parviflora) is indigenous to southeast Asia, including Thailand, and typically contains polymethoxylated flavones. The flavones in $K$. parviflora are reported to have various biological properties. Recently, polymethoxylated flavones of $K$. parviflora (KPMFs) were shown to have potent Sirtuin 1 enzyme-stimulating and anti-glycation activities that led to the suppression of cataract formation. Matrix metalloproteinases (MMPs) are upregulated in several pathologic ocular diseases, including cataracts, and have been established as an attractive target for the prevention and/or treatment of specific cataract phenotypes, such as anterior subcapsular cataract (ASC) and posterior capsular opacification (PCO). In the present study, we investigated the effect of KPMFs on MMP (gelatinase) activity in the human lens epithelial cell line, SRA01/04. We demonstrated that KPMFs inhibited the phorbol ester-induced MMP-9 activity and the mRNA expression through the suppression of mitogen-activated protein kinases (MAPKs) phosphorylation in human lens epithelial cells; 5,7-dimethoxyflavone was found to exert the most potent inhibition, but $3,5,7,4^{\prime}$-tetramethoxyflavone and $3,5,7,3^{\prime}, 4^{\prime}$-pentamethoxyflavone also resulted in considerable inhibition. Our results suggested that the consumption of PMFs isolated from $K$. parviflora, may be an effective strategy to delay the development of cataracts, such as ASC and PCO.

Key words polymethoxylated flavone; Kaempferia parviflora; matrix metalloproteinase-9; lens epithelial cell; cataract; structure-activity relationship

\section{INTRODUCTION}

Cataract is a common worldwide disorder leading to blindness, despite the option of surgery procedure in developed countries. Currently, there are no pharmacological therapy for inhibiting cataract formation. Thus, an understanding of the cellular and molecular mechanisms involved in cataract formation may lead to the development of therapeutic strategies for the prevention or treatment of cataracts.

Matrix metalloproteinases (MMPs) are a family of more than 25 genetically distinct but structurally related zincdependent endopeptidases that act as key regulators of tissue remodeling. MMPs are regulated by their inhibitors, the tissue inhibitors of metalloproteinases (TIMPs), which control enzyme activity and proenzyme activation. MMPs and TIMPs in human eyes have been investigated by several experimental methods, and investigators reported the constitutive expression of multiple MMPs and TIMPs in various ocular tissues, including lenses. ${ }^{1)}$ For example, the use of immunohistochemical and enzyme-linked immunosorbent assay (ELISA) analysis revealed that normal human lenses were found to express MMP-1, MMP-2, MMP-3, MMP-9, TIMP-1, TIMP-2, and TIMP-3. ${ }^{2)}$ In addition, Hodgkinson et al. ${ }^{3)}$ found that membrane-type MMPs (MT-MMPs) genes were strongly expressed in human donor lenses. Of particular interest in the lens are the gelatinases A and B (MMP-2 and MMP-9), which are involved in corneal wound healing processes and cataract formation. In normal lenses, MMP-2 and MMP-9 are expressed at low levels; both are increased when the lens is stressed by radiation, oxidative stress, or transforming growth factor- $\beta$ (TGF- $\beta){ }^{4-6)}$ In addition, evidence has accumulated to show that the expression of specific MMPs including MMP-2 and MMP-9 is induced in a variety of cataract phenotypes, such as anterior subcapsular cataract (ASC) and posterior capsular opacification (PCO). ${ }^{7,8)}$ Recently, Korol et al. ${ }^{9)}$ addressed the individual requirements of MMP-2 and MMP-9 in ASC formation through the use of MMP knockout mice and demonstrated that MMP-9 knockout mice were resistant to TGF- $\beta$-induced ASC formation. These results suggested that the inhibition of MMP activity by MMP inhibitors may be useful for the prevention of specific forms of cataract, such as ASC and PCO.

Many naturally occurring compounds have become candidates for drug development and the subsequent clinical management procedures. Polymethoxylated flavones (PMFs) are found almost exclusively in plants of the Citrus genus. They are of particular interest owing to their broad spectrum of biological activities, which includes antimetastasis, anti-insulin resistance, and growth inhibitory activities. ${ }^{10-12)}$ We have previously reported that nobiletin, a major PMF in citrus fruits, attenuated MMP-9 activity in ocular cells, such as human lens epithelial cells and human retinal Müller cells. ${ }^{13,14)}$ PMFs are also known to be the major active compounds isolated from rhizome of Kaempferia parviflora (K. parviflora), which is a popular food in southeast Asia. The PMFs in $K$. parviflora were reported to have anticancer, steroid $5 \alpha$-reductase inhibitory, and anti-inflammatory, antifungal and anti-gastric ulcer activities. Recently, Nakata et al. ${ }^{15)}$ demonstrated that PMFs 
<smiles>COc1cc(OC)c2c(c1)OC(c1ccccc1)=CC2O</smiles>

5,7-dimethoxyllavone (Compound 1)<smiles>COC1=C(c2ccc(OC)cc2)Oc2cc(OC)cc(OC)c2C1O</smiles>

3,5,7,4'-tetramethoxyflavone (Compound 3)<smiles>COc1cc(OC)c2c(=O)c(OC)c(-c3ccccc3)oc2c1</smiles>

3,5,7-trimethoxyflavone (Compound 2)

Fig. 1. Structure of Polymethoxylated Flavones Isolated from Kaempferia parviflora

of $K$. parviflora, such as $3,5,7,3^{\prime}, 4^{\prime}$-pentamethoxyflavone and 5,7,4'-trimethoxyflavone showed potent Sirtuin 1 (SIRT1) enzyme-stimulating and anti-glycation activities. As SIRT1 enzyme-stimulating and anti-glycation activities are reported to lead to the suppression of cataract formation, ${ }^{16)}$ the intake of polymethoxylated flavones isolated from $K$. parviflora (KPMFs) may be able to delay cataract formation. However, the effect of KPMFs on MMPs activity in lens epithelial cells (LECs) has not been fully elucidated. In the present study, we investigated the effect of PMFs isolated from $K$. parviflora on MMP (gelatinase) activity in human lens epithelial cells.

\section{MATERIALS AND METHODS}

Materials K. parviflora (rhizome) was collected in 2009 in Thailand. Bioactive fractions in $K$. parviflora (rhizome) were separated by partition using water and EtOAc and then repeated column chromatography ${ }^{15)}$ and resulted in the isolation of polymethoxylated flavones as shown in Fig. 1. The human lens epithelial cell line, SRA01/04 was a kind gift from Dr. Nobuhiro Ibaraki (IBARAKI eye clinic, Tochigi, Japan). Antibodies against phospho-extracellular signal-regulated kinase (Erk)1/2 (Thr202/Tyr204), phospho-p38 (Thr180/Tyr182), and phospho-c-Jun N-terminal kinase (JNK) (Thr183/Tyr185) were purchased ed from Cell Signaling Technology, Inc. (Danvers, MA, U.S.A.). Antibodies against TIMP-1, and TIMP-2, and $\beta$-actin and other chemicals were purchased from SigmaAldrich (St. Louis, MO, U.S.A.).

Cell Culture and Treatment SRA01/04 cells were incubated in low glucose $(5 \mathrm{mM})$ Dulbecco's modified Eagle's medium (DMEM) (Invitrogen, Carlsbad, CA, U.S.A.) with $20 \%$ fetal bovine serum (Biowest, Nuaille, France) and penicillin/streptomycin/neomycin antibiotic mixture (Invitrogen) at $37^{\circ} \mathrm{C}$ with $5 \% \mathrm{CO}_{2}$. After reaching sub-confluence, they were treated up to $24 \mathrm{~h}$ with phorbol 12 -myristate 13 -acetate (PMA) in the presence or absence of polymethoxylated flavones isolated from $K$. parviflora in low glucose $(5 \mathrm{mM})$ condition. The media were collected and centrifuged at $1200 \mathrm{rpm}$ for $5 \mathrm{~min}$. The supernatants were used as conditioned media, and the cells were used to prepare the cell fractions.

Gelatin Zymography For gelatin zymography, SRA01/04
Table 1. $\mathrm{IC}_{50}$ Values $(\mu \mathrm{M})$ of Polymethoxylated Flavones Isolated from Kaempferia parviflora for proMMP-9 Production in PMA-Treated SRA01/04 Cells

\begin{tabular}{cc}
\hline \hline Compound & \\
\hline 1 & $08.6 \pm 01.4(n=4)$ \\
2 & $>64(n=4)$ \\
3 & $35.2 \pm 12.0(n=4)$ \\
4 & $25.5 \pm 12.9(n=4)$ \\
\hline
\end{tabular}

were counted and seeded at $1 \times 10^{5}$ cells per well with 6 well plate. After treatment, cell culture supernatants were analyzed for the presence of the secreted MMP-2 and MMP-9 gelatinases using zymography. Quantitative analyses were performed through the Image J software (NIH, Bethesda, MD, U.S.A.) and the $\mathrm{IC}_{50}$ values (shown in Table 1) were estimated from the relative amounts of proMMP-9 at various concentrations $(0.25,1,4,16$, or $64 \mu \mathrm{M})$ of test samples as previously reported. ${ }^{17)}$

Real-Time RT-Quantitative (q)PCR For RT-qPCR, SRA01/04 were counted and seeded at $2 \times 10^{5}$ cells per dish with $60 \mathrm{~mm}$ dish. After treatment, Total RNA was extracted using ISOGEN reagent (Nippon Gene, Toyama, Japan), and first-strand cDNA was synthesized from 500 ng RNA using PrimeScript ${ }^{\circledR}$ RT reagent Kit (TaKaRa Bio Inc, Otsu, Japan) following manufacturer's instruction. The concentrations of synthesized cDNA solution are finally $50 \mathrm{ng} / \mu \mathrm{L}$. The cDNA solution was then used in qPCR reactions to analyze $M M P-9$ expression using SYBR green PCR master mix (TaKaRa Bio) as previously reported. ${ }^{14}$ ) The primers for $\mathrm{qPCR}$ were human MMP-9 (forward 5'-CCC TGG AGA CCT GAG AACCAA-3', reverse 5'-CATCTC TGCCACCCGAGTGTA-3') and human $\beta$-actin (forward 5'-TGGCACCCAGCACAATGAA-3', reverse 5'-CTA AGTCAT AGT CCGCCT AGA AGC A-3'). The standard PCR conditions included denaturation at $95^{\circ} \mathrm{C}$ for $30 \mathrm{~s}$, followed by 40 cycles of extension at $95^{\circ} \mathrm{C}$ for $5 \mathrm{~s}$ and $64^{\circ} \mathrm{C}$ for $34 \mathrm{~s}$. Threshold lines were automatically adjusted to intersect amplification lines in the linear portion of the amplification curves, and the cycles to threshold $(\mathrm{Ct})$ were recorded automatically. Data were normalized to the expression of the endogenous control, $\beta$-actin. The reaction of qPCR was 
[A]

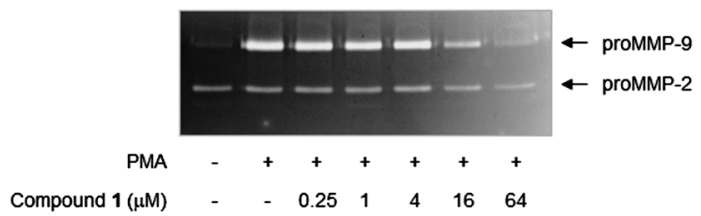

[C]

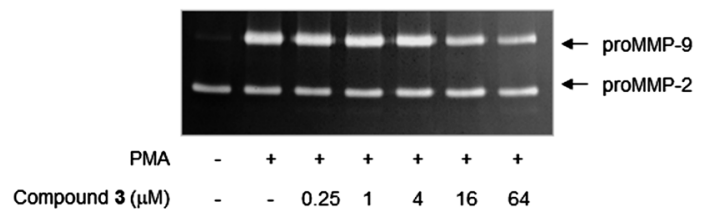

[B]

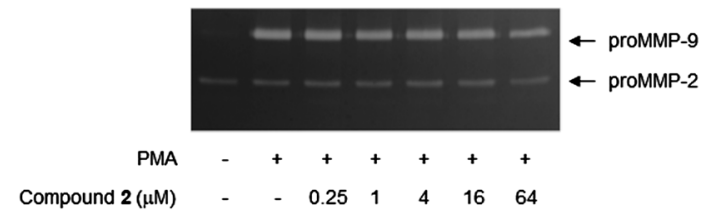

[D]

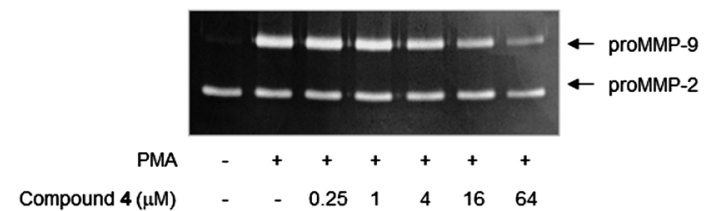

Fig. 2. Effect of Polymethoxylated Flavones Isolated from Kaempferia parviflora on Phorbol Ester-Induced Gelatinase Activity in Human Lens Epithelial Cells

SRA01/04 cell culture supernatants were analyzed for the gelatinase levels of MMP-2 and MMP-9 using gelatin zymography following treatment with 5,7-methoxyflavone (Compound 1) [A], 3,5,7-trimethoxyflavone (Compound 2) [B], 3,5,7,4'-tetramethoxyflavone (Compound 3) [C] or 3,5,7,3',4'-pentamethoxyflavone (Compound 4) [D] in the presence of PMA $(10 \mathrm{nM})$ for $24 \mathrm{~h}$.

performed through Applied Biosystem 7500 Real time PCR System (Life Technologies, Carlsbad, CA, U.S.A.).

Western Blot Analysis For Western blot analysis, SRA01/04 were counted and seeded at $1 \times 10^{5}$ cells per well with 6 well plate. After treatment, the expression of TIMPs in the conditioned media was detected by Western blot analysis using specific rabbit anti-human TIMP-1 antibody (Sigma-Aldrich) and rabbit anti-human TIMP-2 antibody (Sigma-Aldrich) under reducing condition. The dilution rate of TIMP-1 and TIMP-2 antibody is 1000 times. Cell fractions were analyzed for the detection of phospho-Erk1/2, phosphop38 and phospho-JNK, and $\beta$-actin by Western blot analysis using specific rabbit antibodies against phospho-Erk1/2 (Thr202/Tyr204), phospho-p38 (Thr180/Tyr182), phospho-JNK (Thr183/Tyr185) (Cell Signaling Technology) and $\beta$-actin (Sigma-Aldrich). The dilution rate of phosphor-mitogen-activated protein kinases (MAPKs) and $\beta$-actin antibody are 1000 and 10000 times, respectively. $\beta$-Actin is used as a loading control to confirm equal loading protein on each lane. Quantitative analyses were performed using the Image J software (NIH), as previously reported. ${ }^{14)}$ The relative amounts of the immunoreactive proteins were quantified by densitometric scanning using an Amersham Imager 680 (GE Healthcare). Protein concentrations in conditioned media were determined with BCA protein analysis kit (Thermoscientific, Rockford, IL, U.S.A.).

Statistical Analysis Data are expressed as the mean \pm standard deviation (S.D.) and analyzed using Student's $t$-test. For all analysis, $p<0.05$ was considered to indicate a statistically significant difference.

\section{RESULTS}

Effects of Polymethoxylated Flavones Isolated from $K$. parviflora on Gelatinase Activity The activation of protein kinase $\mathrm{C}(\mathrm{PKC})$ is reported to be involved in cataract development through the inhibition of gap junction intercellular communication (GJIC), which enables the regulation of waste and nutrient transport to maintain lens transparency ${ }^{18)}$; in addition, GJIC dysfunction is reported to be involved in cataractogenesis. ${ }^{19)}$ Long et $a l .{ }^{18)}$ provided evidence that PMA, known as a PKC activator, treatment at range of concentration between $2 \mathrm{nM}$ and $200 \mathrm{nM}$ resulted in a significant reduction in GJIC in canine lens epithelial cells. At the range of these concentrations, treatment of PMA $(10 \mathrm{nM})$ is reported to augment MMP-9 activity in several cells such as human fibrosarcoma HT1080 cells ${ }^{10)}$ and human retinal Müller cells. ${ }^{20)}$ We had previously reported that treatment of PMA $(10 \mathrm{nM})$ augmented MMP-9 activity in the human lens epithelial cell line, SRA01/04. ${ }^{17)}$ Therefore, we examined the effect of KPMFs on the PMA-mediated activities of gelatinase through the analysis of conditioned media from KPMFs-treated cells by gelatin zymography. As shown in Fig. 2, although the strength of inhibitory activity was different, all KPMFs were found to reduce PMA-induced MMP-9 activity in SRA01/04 cells in a dose-dependent manner. Among the KPMFs, 5,7-dimethoxyflavone showed the most potent inhibition of PMA-stimulated MMP-9 activity $\left(\mathrm{IC}_{50}: 8.6 \mu \mathrm{M}\right)$, followed by $3,5,7,3^{\prime}, 4^{\prime}$-pentamethoxyflavone $\left(\mathrm{IC}_{50}: 25.5 \mu \mathrm{M}\right)$ and then $3,5,7,4^{\prime}$-tetramethoxyflavone $\left(\mathrm{IC}_{50}: 35.2 \mu \mathrm{M}\right)$. 3,5,7-Trimethoxyflavone also inhibited MMP-9 activity, but to a lesser extent than other KPMFs $\left(\mathrm{IC}_{50}:>64 \mu \mathrm{M}\right)$. However, KPMFs did not affect MMP-2 activity in PMA-stimulated SRA01/04 cells. As a result of statistical analysis, $\mathrm{IC}_{50}$ values of 5,7-dimethoxyflavone was found to be significantly lower than 3,5,7,4'-tetramethoxyflavone $(p<0.05)$. On the other hand, $\mathrm{IC}_{50}$ values between 5,7-dimethoxyflavone and 3,5,7,3',4'-pentamethoxyflavone did not show no significant difference.

Effects of Polymethoxylated Flavones Isolated from $K$. parviflora on MMP-9 mRNA Expression To clarify the downregulation of MMP-9 activity by KPMFs, we further examined the effect of KPMFs on the MMP-9 mRNA expression by using RT-qPCR analysis. As shown in Fig. 3, PMA augmented the mRNA expression of MMP-9 and KPMFs except 3,5,7-trimethoxyflavone significantly reduced the PMA-induced MMP-9 mRNA expression in SRA01/04 cells $(p<0.05)$. 5,7-Dimethoxyflavone and 3,5,7,3',4'-pentamethoxy- 
flavone had comparable inhibitory action on the PMA-induced MMP-9 mRNA expression. On the other hand, 3,5,7-tetramethoxyflavone and 3,5,7,4'-tetramethoxyflavone were less active against PMA-induced MMP-9 mRNA expression compared to 5,7-dimethoxyflavone $(p<0.05)$. Among KPMFs, 5,7-dimethoxyflavone showed the most potent inhibition of PMA-stimulated MMP-9 activity and MMP-9 mRNA expression significantly different from 3,5,7,4'-tetramethoxyflavone $(p<0.05)$. The suppression strength on MMP-9 mRNA expression by 5,7-dimethoxyflavone was found to be significantly higher than $3,5,7,3^{\prime}, 4^{\prime}$-pentamethoxyflavone, and $\mathrm{IC}_{50}$ values of 5,7-dimethoxyflavone tended to be lower than 3,5,7,3',4'-pentamethoxyflavone although the difference was not significance. On the whole, the strength of inhibitory action on the MMP-9

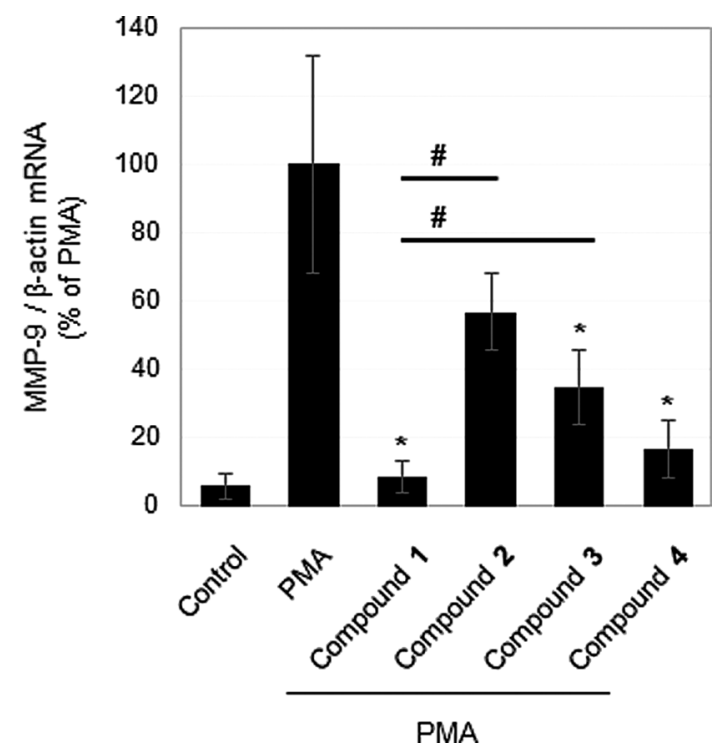

Fig. 3. Effect of Polymethoxylated Flavones Isolated from Kaempferia parviflora on Phorbol Ester-Induced MMP-9 mRNA Expression in Human Lens Epithelial Cells

Total RNA isolated from SRA01/04 cells were analyzed for the mRNA expression of MMP-9 and $\beta$-actin using RT-qPCR following treatment with polymethoxylated flavones $(64 \mu \mathrm{M})$ isolated from Kaempferia parviflora in the presence of PMA $(10 \mathrm{nM})$ for $24 \mathrm{~h}$. Data are shown as means \pm S.D. of four independent experiments. *Significantly different from PMA-treated cells $(p<0.05)$. "Significantly different from 5,7-dimethoxyflavone-treated cells $(p<0.05)$.
mRNA expression by KPMFs tended to correlate with that of the inhibitory action on MMP-9 activity. These results suggested that the inhibitory effect of KPMFs on MMP-9 activity results in a reduction of MMP-9 mRNA expression.

Effects of Polymethoxylated Flavones Isolated from K. parviflora on MAPKs Phosphorylation To examine the molecular mechanisms underlying the MMP regulation by KPMFs, we examined the effects of KPMFs on the phospho-MAPKs expression in PMA-stimulated SRA01/04 cells by using Western blotting. As shown in Fig. 4, PMA increased the phospho-MAPKs (Erk1/2, p38, and JNK) in SRA01/04 cells. 5,7-Dimethoxyflavone significantly reduced the phosphorylation of Erk1/2 $(p<0.01)$, which was different to the effects of the other three KPMFs. In addition, 5,7-dimethoxyflavone significantly suppressed the PMA-induced phosphorylation of p38 $(p<0.001)$ and JNK $(p<0.05)$. Both $3,5,7,4^{\prime}$-tetramethoxyflavone and $3,5,7,3^{\prime}, 4^{\prime}$-pentamethoxyflavone were also found to significantly inhibit the PMA-induced phosphorylation of p38 and JNK, comparable with 5,7-dime-

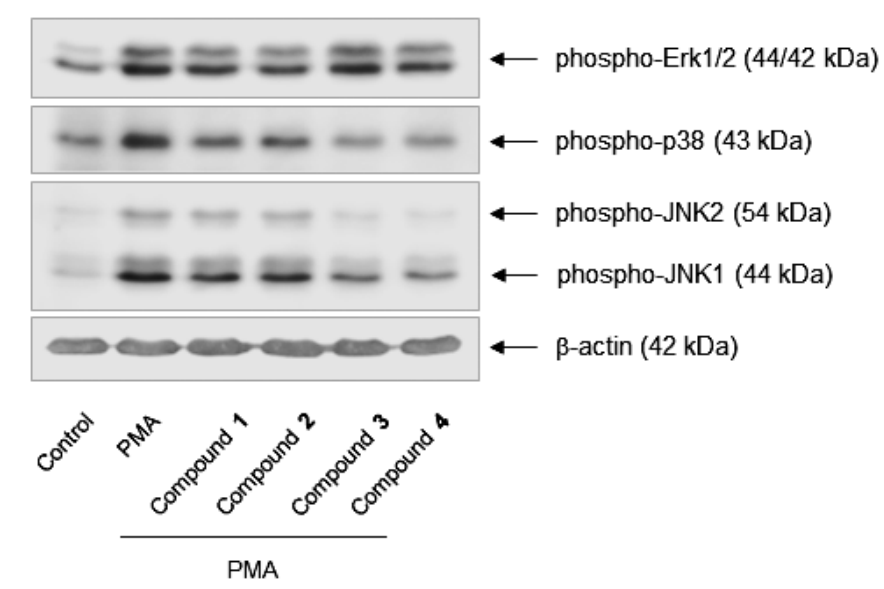

Fig. 4. Effect of Polymethoxylated Flavones Isolated from Kaempferia parviflora on Phosphorylated MAPKs in Phorbol Ester-Treated Human Lens Epithelial Cells

Western blot analysis revealed phospho-MAPKs (Erk1/2, p38, and JNK1/2) or $\beta$-actin. SRA01/04 cells were pretreated with polymethoxylated flavones isolated $(64 \mu \mathrm{M})$ from Kaempferia parviflora for $2 \mathrm{~h}$, and then treated with PMA (10nM) for another $20 \mathrm{~min}$.
[A]

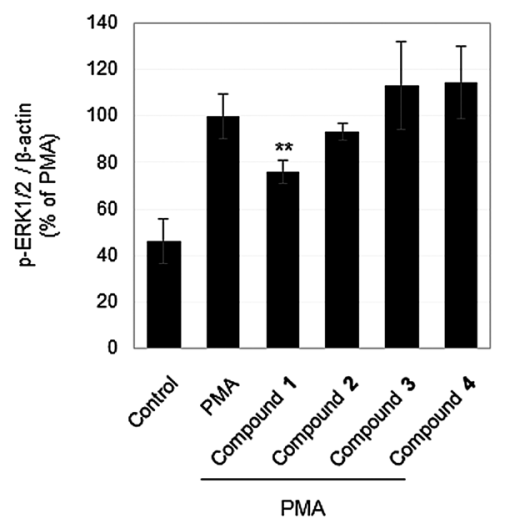

$[B]$

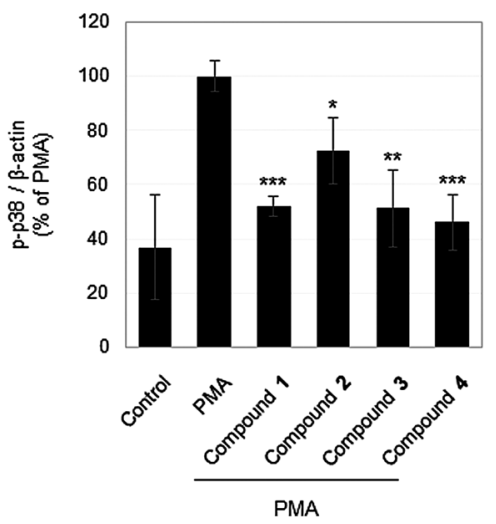

[C]

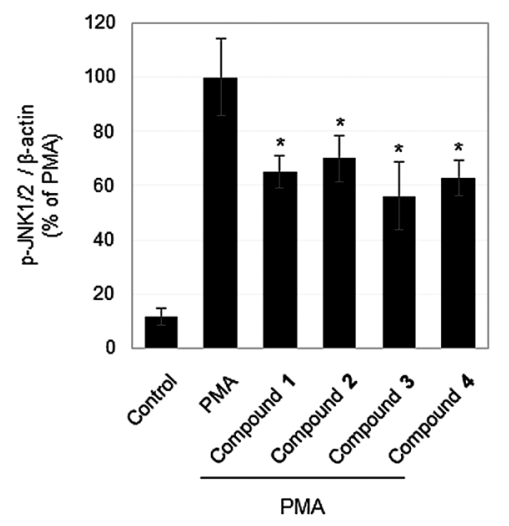

Fig. 5. Inhibitory Action against MAPKs Phosphorylation by Polymethoxylated Flavones Isolated from Kaempferia parviflora in Phorbol Estertreated Human Lens Epithelial Cells

Western blot analysis revealed phospho-Erk1/2 [A], phospho-p38 [B], phospho-JNK1/2 [C] or $\beta$-actin. SRA01/04 cells were pretreated with polymethoxylated flavones isolated $(64 \mu \mathrm{M})$ from Kaempferia parviflora for $2 \mathrm{~h}$, and then treated with PMA $(10 \mathrm{nM})$ for another 20 min. Data are shown as means \pm S.D. of four independent experiments. $* * *$ and $* * *$ significantly different from PMA-treated cells $(p<0.05,0.01$ and 0.001 , respectively). 


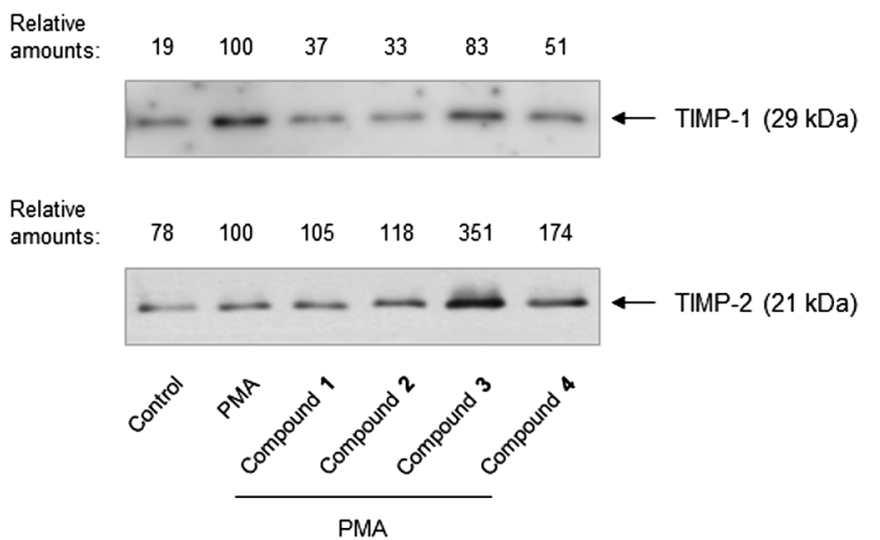

Fig. 6. Effect of Polymethoxylated Flavones Isolated from Kaempferia parviflora on TIMPs Production in Phorbol Ester-Treated Human Lens Epithelial Cells

SRA01/04 cell culture supernatants were analyzed for the production of TIMP-1 and TIMP-2 using Western blot analysis following treatment with polymethoxylated flavones isolated $(64 \mu \mathrm{M})$ from Kaempferia parviflora in the presence of PMA (10 $\mathrm{nM})$ for $24 \mathrm{~h}$.

thoxyflavone. However, the inhibitory effect of 3,5,7-trimethoxyflavone on the PMA-induced phosphorylation of p38 and JNK by tended to be weaker than that of the other three KPMFs (Fig. 5).

Effects of Polymethoxylated Flavones Isolated from $K$. parviflora on TIMPs Production MMPs are proteolytic enzymes controlled by a family of natural antagonists, TIMPs. TIMP-1 exhibits a greater inhibition for MMP-9 compared to other MMPs, and TIMP-2 strongly binds to proMMP-2 to form a complex that is significant in the cell surface activation of proMMP-2. Finally, we examined the effects of KPMFs on the TIMP-1 and TIMP-2 production in PMA-stimulated SRA01/04 cells. As shown in Fig. 6, we found that SRA01/04 cells constitutively produce both TIMP-1 and TIMP-2. Treatment with PMA remarkedly augmented TIMP-1 production. Among the KPMFs, 5,7-dimethoxyflavone, 3,5,7-trimethoxyflavone, and 3,5,7,3', $4^{\prime}$-pentamethoxyflavone were found to inhibit PMA-induced TIMP-1 production, although the activity of 3,5,7,4'-tetramethoxyflavone was lower. These results suggested that KPMFs may not exert inhibitory effect against MMP-9 activity through the augmentation of TIMP-1 production in PMA-stimulated SRA01/04 cells. TIMP-2 production was slightly enhanced by PMA, and 3,5,7,4'-tetramethoxyflavone and 3,5,7,3',4'-pentamethoxyflavone remarkedly augmented the production of TIMP-2 in PMA-stimulated SRA01/04 cells. However, 5,7-dimethoxyflavone and 3,5,7-trimethoxyflavone did not affect the PMA-stimulated TIMP-2 production. To ensure the equal loading between each sample, we examined the total protein concentration in culture media. We demonstrated that there is no significant different between protein concentrations in culture media collected from PMA and/or KPMFs-treated cells (Table 2). In addition, we performed Ponceau $\mathrm{S}$ staining using the membranes prepared from conditioned media after treatment of KPMFs in PMAstimulated SRA01/04 cells. As a result, the expressions of Ponceau S-binding protein on membrane were found to be approximately same between each sample (see Supplementary Materials). We concluded that these results could guarantee the equal loading and transfer efficiency between each sample for Western blotting of TIMPs.
Table 2. Protein Concentrations of the Conditioned Medium of MIO-M1 Measured by BCA Analysis for Each Preparation

\begin{tabular}{lcc}
\hline \hline Treatment & $\begin{array}{c}\text { PMA or compound } \\
\text { concentration }\end{array}$ & $\begin{array}{c}\text { Protein concentration } \\
(\mathrm{mg} / \mathrm{mL})\end{array}$ \\
\hline Control & & $0.95 \pm 0.02(n=4)$ \\
PMA & $10 \mathrm{nM}$ & $0.89 \pm 0.02(n=4)$ \\
PMA $(10 \mathrm{nM})+$ compound $\mathbf{1}$ & $64 \mathrm{mM}$ & $0.92 \pm 0.03(n=4)$ \\
PMA $(10 \mathrm{nM})+$ compound $\mathbf{2}$ & $64 \mathrm{mM}$ & $0.91 \pm 0.02(n=4)$ \\
PMA $(10 \mathrm{nM})+$ compound 3 & $64 \mathrm{mM}$ & $0.95 \pm 0.03(n=4)$ \\
PMA (10nM) + compound 4 & $64 \mathrm{mM}$ & $1.00 \pm 0.01(n=4)$ \\
\hline
\end{tabular}

\section{DISCUSSION}

Proteases, such as the MMPs, play an important role in lens development and cataract formation, and many researchers have focused their attention on fibrotic cataracts, ASC and PCO. Among MMPs, MMP-9 expression is more critical in cataract formation, such as ASC and PCO, through the induction of proteolytic cleavage of latent $\operatorname{TGF} \beta$ and E-cadherin, which leads to the epithelial-mesenchymal transition (EMT) associated with lens opacity. ${ }^{21,22)}$ In addition, Alapure et al. ${ }^{23)}$ evaluated the level of MMP-9 activity in LECs derived from different types of age-related cataracts in patients and found that the highest MMP-9 activity in patient-derived LECs was in cortical cataracts. Several reports also suggested that MMPs have potential causative roles in age-related cataract formation, probably through the induction of intracellular $\beta$-crystallin aggregation and the shedding of growth factor receptors. ${ }^{24,25)}$ Therefore, MMP inhibitors may be an effective strategy for the prevention or treatment of not only fibrotic cataracts such as ASC and PCO, but also age-related cortical cataracts. In this study, KPMFs inhibited PMA-mediated MMP-9 mRNA expression, which leads to the MMP-9 activity in LECs.

The regulation of the MAPKs is a prominent mechanism for the regulation of that MMP expression. In the present study, we observed that PMA, a PKC activator, augmented MAPKs phosphorylation in lens epithelial cells. In addition, specific MAPK inhibitors of ERK1/2 (PD98059), p38 (SB203580), and JNK (SP600125) suppressed the PMAinduced MMP-9 mRNA expression following the inhibition of MMP-9 activity in SRA01/04 cells (see Supplementary Materials). We also demonstrated that all KPMFs inhibited the PMA-induced phosphorylation of p38 and JNK in SRA01/04 cells. These results suggested that the regulation of MMP-9 mRNA expression by KPMFs occurred through the PKC/p38 and $\mathrm{PKC} / \mathrm{JNK}$ pathways in LECs. Interestingly, our results demonstrated that the inhibition of the PKC/ERK1/2 pathway led to an increase in the inhibitory action of 5,7-dimethoxyflavone on the mRNA expression of MMP-9. ERK1/2, a type of MAPK, has been implicated as an important component in many signaling systems containing polymethoxylated flavones involved in various biological functions, such as the differentiation and lipolysis of adipocytes, neurotrophic action, and MMP expression. ${ }^{14,26,27)}$ In lens epithelial cells, ERK1/2 was reported to be involved in the TGF $\beta$-induced $\mathrm{EMT}^{28)}$; therefore, 5,7-dimethoxyflavone may inhibit the EMT in TGF $\beta$ treated LECs, and lead to the visual impairments observed in ASC and PCO. The relationship between the expression of MMP-9 and the EMT, and the regulation of this by KPMFs, 
should be investigated further in the future.

The number and position of the methoxy groups of polymethoxylated flavones are reported to be closely associated with their biological activity. In the present study, we found that 5,7-dimethoxyflavone showed the most potent inhibitory effects on MMP-9 activity and mRNA expression compared with other KPMFs. On the other hand, our results also demonstrated that the addition of the methoxy group at the 3-position of the C-ring tended to lead to a significant reduction in the inhibitory effects on MMP-9 activity and mRNA expression by comparing the strength of inhibitory action between 5,7-dimethoxyflavone and 3,5,7-trimethoxyflavone or 3,5,7,4'-tetramethoxyflavone. However, the molecular mechanism of this reduction in the inhibitory effects on MMP-9 mRNA expression by KPMFs with a methoxy group at the 3-position of the C-ring remains to be fully elucidated. In this study, 3,5,7-trimethoxyflavone showed the weakest inhibitory action against MMP-9 mRNA expression but suppressed p38 and JNK phosphorylation to a similar extent as 3,5,7,4'-tetramethoxyflavone and 3,5,7,3',4'-pentamethoxyflavone in PMA-stimulated SRA01/04 cells. These results suggested that another signal transduction pathway may be associated with the additional inhibitory effects of 3,5,7,4'-tetramethoxyflavone and 3,5,7,3',4'-pentamethoxyflavone on MMP-9 mRNA expression. Taiyab et $a .^{29)}$ recently demonstrated that $\beta$-catenin/CBPdependent signaling was involved in the regulation of MMP-9 expression in rat lens epithelial explants. The investigation to clarify the effect of KPMFs with a methoxy group at the 3-position of the $\mathrm{C}$-ring on $\beta$-catenin/CBP-dependent signaling is a challenge for the future. Furthermore, Nakata et al. ${ }^{15)}$ demonstrated that a 4'-methoxy moiety on the B-ring in KPMFs was essential for SIRT1 enzyme-stimulating and anti-glycation activities. In the present study, the KPMFs with 4'-methoxy moiety on B-ring in KPMFs showed significant inhibition on PMA-induced MMP-9 mRNA expression. Therefore, the presence of a $4^{\prime}$-methoxy group may be necessarily required for not only SIRT1 enzyme-stimulating and anti-glycation activities but also the inhibition of MMP-9 activity. Yenjai et al. ${ }^{30)}$ structurally modified 5,7-dimethoxyflavone and synthesized more polar derivatives, such as phenolic or oxime compounds, which were shown to have greater antifungal activity against Candida albicans than their parent compound, 5,7-dimethoxyflavone. In future, it should be investigated if these structural modifications of 5,7-dimethoxyflavone affect the inhibitory effect against MMP-9 activity.

In conclusion, MMPs may be a promising target that exerts multiple actions on specific cataract phenotypes, such as ASC and PCO. In this study, KPMFs inhibited MMP-9 activity through the suppression of MAPKs-mediated MMP-9 mRNA expression. Therefore, the dietary intake of KPMFs may have therapeutic value for the prevention or treatment of fibrotic cataracts such as ASC and PCO. This study has provided novel findings related to the specific chemical structures of KPMFs that enhance the inhibitory effects on MMP-9 activity; 5,7-dimethoxyflavone was found to be the most effective for the inhibition of MMP-9 activity. The delivery of KPMFs to lens presents challenges in the prevention or treatment of cataracts, and the delivery of KPMFs directly to the LECs from the intraocular lens may offer a very promising solution to reduce the incidence of secondary cataract formation. Further insights into the bioavailability of KPMFs are necessary to evaluate their pharmacological action within target tissues. The dietary intake of KPMFs may delay the cataract formation.

Acknowledgments We thank Mr. Kazuya Matsumoto, Ms. Aoi Hirata, Ms. Chizuru Fukui and Ms. Saori Watanabe for research assistance.

Conflict of Interest Yoshiki Miyata has received a research Grant from Tokiwa Phytochemical Co., Ltd.

Supplementary Materials The online version of this article contains supplementary materials.

\section{REFERENCES}

1) Sivak JM, Fini ME. MMPs in the eye: emerging roles for matrix metalloproteinases in ocular physiology. Prog. Retin. Eye Res., 21, 1-14 (2002)

2) Sachdev NH, Di Girolamo N, Nolan TM, McCluskey PJ, Wakefield D, Coroneo MT. Matrix metalloproteinases and tissue inhibitors of matrix metalloproteinases in the human lens: implications for cortical cataract formation. Invest. Ophthalmol. Vis. Sci., 45, 4075-4082 (2004).

3) Hodgkinson LM, Duncan G, Wang L, Pennington CJ, Edwards DR, Wormstone IM. MMP and TIMP expression in quiescent, dividing, and differentiating human lens cells. Invest. Ophthalmol. Vis. Sci., 48, 4192-4199 (2007).

4) Chang PY, Bjornstad KA, Rosen CJ, Lin S, Blakely EA. Particle radiation alters expression of matrix metalloproteases resulting in ECM remodeling in human lens cells. Radiat. Environ. Biophys., 46, 187-194 (2007).

5) Tamiya S, Wormstone IM, Marcantonio JM, Gavrilovic J, Duncan $\mathrm{G}$. Induction of matrix metalloproteinases 2 and 9 following stress to the lens. Exp. Eye Res., 71, 591-597 (2000).

6) Wormstone IM, Tamiya S, Anderson I, Duncan G. TGF-beta2induced matrix modification and cell transdifferentiation in the human lens capsular bag. Invest. Ophthalmol. Vis. Sci., 43, 23012308 (2002).

7) West-Mays JA, Pino G. Matrix metalloproteinases as mediators of primary and secondary cataracts. Expert Rev. Ophthalmol., 2, 931-938 (2007)

8) Dwivedi DJ, Pino G, Banh A, Nathu Z, Howchin D, Margetts P, Sivak JG, West-Mays JA. Matrix metalloproteinase inhibitors suppress transforming growth factor-beta-induced subcapsular cataract formation. Am. J. Pathol., 68, 69-79 (2006).

9) Korol A, Pino G, Dwivedi D, Robertson JV, Deschamps PA, WestMays JA. Matrix metalloproteinase-9-null mice are resistant to TGF- $\beta$-induced anterior subcapsular cataract formation. Am. J. Pathol., 184, 2001-2012 (2014).

10) Miyata $Y$, Sato $T$, Yano M, Ito A. Activation of protein kinase $C$ betaII/epsilon-c-Jun NH2-terminal kinase pathway and inhibition of mitogen-activated protein/extracellular signal-regulated kinase $1 / 2$ phosphorylation in antitumor invasive activity induced by the polymethoxy flavonoid, nobiletin. Mol. Cancer Ther., 3, 839-847 (2004).

11) Miyata $Y$, Tanaka H, Shimada A, Sato T, Ito A, Yamanouchi T, Kosano H. Regulation of adipocytokine secretion and adipocyte hypertrophy by polymethoxyflavonoids, nobiletin and tangeretin. Life Sci., 88, 613-618 (2011).

12) Miyata Y, Oshitari $T$, Okuyama $Y$, Shimada A, Takahashi $\mathrm{H}$, Natsugari H, Kosano H. Polymethoxyflavones as agents that prevent formation of cataract: nobiletin congeners show potent growth inhibitory effects in human lens epithelial cells. Bioorg. Med. Chem. Lett., 23, 183-187 (2013).

13) Oshitari T, Okuyama Y, Miyata Y, Kosano H, Takahashi H, Natsug- 
ari H. Nobiletin metabolites: synthesis and inhibitory activity against matrix metalloproteinase-9 production. Bioorg. Med. Chem. Lett., 21, 4540-4544 (2011).

14) Miyata $Y$, Nagase $T$, Katsura $Y$, Takahashi $H$, Natsugari H, Oshitari $\mathrm{T}$, Kosano $\mathrm{H}$. In vitro studies on nobiletin isolated from citrus plants and the bioactive metabolites, inhibitory action against gelatinase enzymatic activity and the molecular mechanisms in human retinal Müller cell line. Biomed. Pharmacother., 93, 70-80 (2017).

15) Nakata A, Koike Y, Matsui H, Shimadad T, Aburada M, Yang J. Potent SIRT1 enzyme-stimulating and anti-glycation activities of polymethoxyflavonoids from Kaempferia parviflora. Nat. Prod. Commun., 9, 1291-1294 (2014).

16) Mimura T, Kaji Y, Noma H, Funatsu H, Okamoto S. The role of SIRT1 in ocular aging. Exp. Eye Res., 116, 17-26 (2013).

17) Oshitari T, Okuyama Y, Miyata Y, Kosano H, Takahashi H, Natsugari H. B-Ring-modified and/or 5-demethylated nobiletin congeners: inhibitory activity against pro-MMP-9 production. Bioorg. Med. Chem., 19, 7085-7092 (2011).

18) Long AC, Colitz CM, Bomser JA. Regulation of gap junction intercellular communication in primary canine lens epithelial cells: role of protein kinase C. Curr. Eye Res., 32, 223-231 (2007).

19) Gao J, Sun X, Martinez-Wittinghan FJ, Gong X, White TW, Mathias RT. Connections between connexins, calcium, and cataracts in the lens. J. Gen. Physiol., 124, 289-300 (2004).

20) Miyata Y, Kase M, Sugita Y, Shimada A, Nagase T, Katsura Y, Kosano H. Protein kinase C-mediated regulation of matrix metalloproteinase and tissue inhibitor of metalloproteinase production in a human retinal müller cells. Curr. Eye Res., 37, 842-849 (2012).

21) Dallas SL, Rosser JL, Mundy GR, Bonewald LF. Proteolysis of latent transforming growth factor-beta (TGF-beta)-binding protein-1 by osteoclasts. A cellular mechanism for release of TGF-beta from bone matrix. J. Biol. Chem., 277, 21352-21360 (2002).
22) Imhof BA, Vollmers HP, Goodman SL, Birchmeier W. Cell-cell interaction and polarity of epithelial cells: specific perturbation using a monoclonal antibody. Cell, 35, 667-675 (1983).

23) Alapure BV, Praveen MR, Gajjar D, Vasavada AR, Rajkumar S, Johar K. Matrix metalloproteinase-9 activity in human lens epitheial cells of cortical, posterior subcapsular, and nuclear cataracts. $J$. Cataract Refract. Surg., 34, 2063-2067 (2008).

24) Trivedi VD, Raman B, Ramakrishna T, Rao CM. Detection and assay of proteases using calf lens beta-crystallin aggregate as substrate. J. Biochem. Biophys. Methods, 40, 49-55 (1999).

25) Imai K, Hiramatsu A, Fukushima D, Pierschbacher MD, Okada Y. Degradation of decorin by matrix metalloproteinases: identification of the cleavage sites, kinetic analyses and transforming growth factor-beta1 release. Biochem. J., 322, 809-814 (1997).

26) Saito T, Abe D, Sekiya K. Nobiletin enhances differentiation and lipolysis of 3T3-L1 adipocytes. Biochem. Biophys. Res. Commun., 357, 371-376 (2007).

27) Nagase H, Yamakuni T, Matsuzaki K, Maruyama Y, Kasahara J, Hinohara Y, Kondo S, Mimaki Y, Sashida Y, Tank AW, Fukunaga $\mathrm{K}$, Ohizumi Y. Mechanism of neurotrophic action of nobiletin in PC12D cells. Biochemistry, 44, 13683-13691 (2005).

28) Chen X, Ye S, Xiao W, Wang W, Luo L, Liu Y. ERK1/2 pathway mediates epithelial-mesenchymal transition by cross-interacting with TGF $\beta /$ Smad and Jagged/Notch signaling pathways in lens epithelial cells. Int. J. Mol. Med., 33, 1664-1670 (2014).

29) Taiyab A, Korol A, Deschamps PA, West-Mays JA. $\beta$-Catenin/CBPdependent signaling regulates TGF- $\beta$-induced epithelial to mesenchymal transition of lens epithelial cells. Invest. Ophthalmol. Vis. Sci., 57, 5736-5747 (2016).

30) Yenjai C, Wanich S, Pitchuanchom S, Sripanidkulchai B. Structural modification of 5,7-dimethoxyflavone from Kaempferia parviflora and biological activities. Arch. Pharm. Res., 32, 1179-1184 (2009). 